Article

\title{
Comparative Transcriptome Analysis of Ampelopsis megalophylla for Identifying Genes Involved in Flavonoid Biosynthesis and Accumulation during Different Seasons
}

\author{
Min Yang ${ }^{\dagger}$, Peina Zhou ${ }^{+}(\mathbb{D}$, Chun Gui, Guozheng Da, Ling Gong * and Xiuqiao Zhang * \\ College of Pharmacy, Hubei University of Chinese Medicine, Wuhan 430065, China; \\ yangminzj@ntu.edu.cn (M.Y.); zhoupeina@163.com (P.Z.); gc19890703@163.com (C.C.); daziwy@163.com (G.D.) \\ * Correspondence: gl1224@163.com (L.G.); qiaoxzh2000@163.com (X.Z.); \\ Tel.: +86-180-6244-2630 (L.G.); +86-138-7139-2318 (X.Z.) \\ + These authors contributed equally to this work.
}

Received: 3 March 2019; Accepted: 28 March 2019; Published: 1 April 2019

check for updates

\begin{abstract}
Ampelopsis megalophylla is an important species used in Chinese folk medicine. Flavonoids, the most important active components of plants, greatly determine the quality of A. megalophylla. However, biosynthesis of flavonoids at the molecular and genetic levels in A. megalophylla is not well understood. In this study, we performed chemical analysis and transcriptome analysis of A. megalophylla in different seasons (i.e., May, August, and October). Accumulation of flavonoids was higher in May than in the other two months. Genes involved in the flavonoid biosynthesis pathway, such as chalcone synthase, anthocyanidin synthase, flavanone 3-hydroxylase, flavonoid- $3^{\prime}, 5^{\prime}$-hydroxylase, caffeoyl-CoA O-methyltransferase, dihydroflavonol 4-reductase, 4-coumarate-CoA ligase, phenylalanine ammonia-lyase, cinnamate 4-hydroxylase, flavonoid $3^{\prime}$-monooxygenase, shikimate $O$-hydroxycinnamoyltransferase, and leucoanthocyanidin reductase, were identified based on transcriptome data. Fifty ATP binding cassette (ABC) transporter, nine SNARE, forty-nine GST, and eighty-four glycosyltransferases unigenes related to flavonoid transport and biomodification were also found. Moreover, seventy-eight cytochrome P450s and multiple transcription factors (five MYB, two bHLH, and three WD40 family genes) may be associated with the regulation of the flavonoid biosynthesis process. These results provide insights into the molecular processes of flavonoid biosynthesis in A. megalophylla and offer a significant resource for the application of genetic engineering in developing varieties with improved quality.
\end{abstract}

Keywords: Ampelopsis megalophylla; flavonoid biosynthesis; flavonoid accumulation; transcriptome

\section{Introduction}

Ampelopsis megalophylla Diels et Gilg, which belongs to the family Vitaceae, is a highly prized folk medicinal herb in the Western parts of Hubei Province in China, where it is native. The dried leaves of A. megalophylla, also called "Meicha", are often consumed as herbal tea and have been used for the treatment of hypertension in clinical settings. Modern pharmacology studies indicate that A. megalophylla has broad pharmacological functions, including anti-hepatitis B virus (HBV), antimicrobial, antioxidant, and antitumor properties [1-4]. These properties have been attributed to the characteristic secondary metabolites of $A$. megalophylla, including flavonoids such as ampelopsin (dihydromyricetin), myricetin, and myricitrin $[5,6]$.

In recent years, a sharp decline has occurred in the natural populations of A. megalophylla, owing to intensive harvesting of this valuable medicinal plant. It is important to develop methods to artificially 
cultivate this species in order to protect wild medicinal plant populations and resolve the shortage of this resource. Thus, there is an urgent need to conduct studies focused on improving the quality of $A$. megalophylla grown under artificial conditions. It is well-known that the quality of traditional Chinese herbs depends on their intrinsic active compounds, and the season is known as a crucial factor that influences the formation and accumulation of secondary metabolites in plants. It has been reported that the bud period of $A$. megalophylla begins in April, the defoliating period occurs in November, and the best harvest time for medicinal purposes is May, when the highest level of flavonoids occurs [7]. Understanding the regulation mechanism of formation and accumulation of flavonoids during different seasons may greatly facilitate the development of $A$. megalophylla with high quality.

Flavonoids are a large group of secondary metabolites and have attracted much attention worldwide due to their potential value in the pharmaceutical and nutraceutical industries $[8,9]$. Studies on the flavonoid biosynthesis pathway of plants have been performed, and it is generally believed that genes associated with the flavonoid synthesis pathway are mainly divided into two categories [10]: Structural genes and regulatory genes. The regulatory genes category includes the MYB-bHLH-WD40 transcription factors. In addition, glycosyltransferases and cytochrome P450s also contribute to flavonoid regulation in plants [11]. However, genes involved in flavonoid biosynthesis in A. megalophylla have not been reported, largely due to non-availability of the reference genome sequence and lack of genomic information for this species. In the present study, to better understand the molecular mechanisms of flavonoid biosynthesis during different seasons in A. megalophylla, we determined quantitative differences of flavonoids in A. megalophylla samples collected at different times of the year. We also performed constituent and transcriptome analysis to reveal the genes associated with flavonoid biosynthesis and accumulation.

\section{Results}

\subsection{Determination of Flavonoids in A. megalophylla}

HPLC analysis was performed to detect dihydromyricetin, myricitrin, quercitrin, and myricetin in leaves of A. megalophylla harvested during different seasons (May, August, and October). As shown in Table 1, concentration of these four flavonoids varied between samples collected from the three different months. Dihydromyricetin, myricitrin, and quercitrin contents were higher in May than those in the August and October (Figure 1), whereas myricetin contents were highest in August.

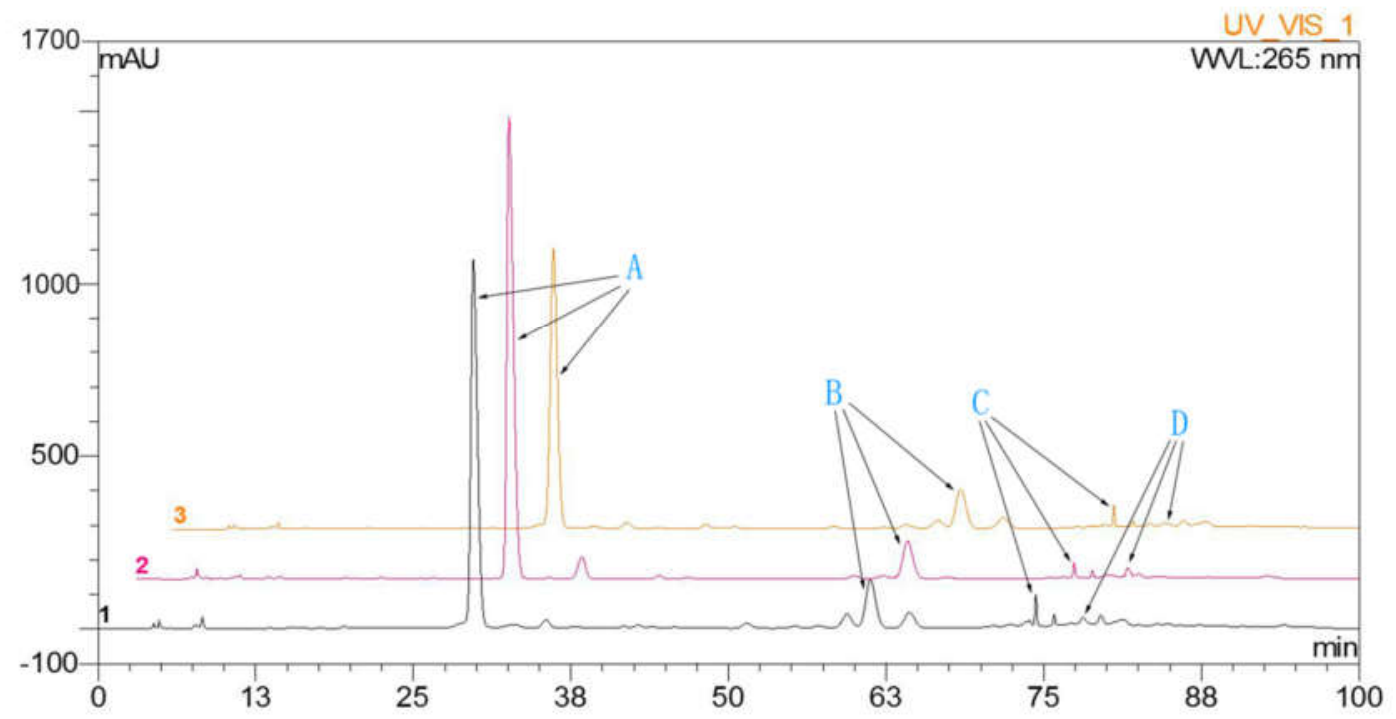

Figure 1. The HPLC chromatographic: A, dihydromyricetin; B, myricitrin; C, quercitrin; D, myricetin. 
Table 1. The content of active component in Ampelopsis megalophylla (mg/g, $\bar{\chi}+\mathrm{D}, n=3)$.

\begin{tabular}{ccccc}
\hline Samples & Dihydromyricetin & Myricitrin & Quercitrin & Myricetin \\
\hline May & $331.226 \pm 1.976$ & $20.889 \pm 0.518$ & $2.334 \pm 0.025$ & $2.455 \pm 0.353$ \\
August & $277.761 \pm 4.908$ & $15.836 \pm 0.127$ & $1.202 \pm 0.0319$ & $2.843 \pm 0.482$ \\
October & $220.605 \pm 1.652$ & $18.308 \pm 0.328$ & $1.659 \pm 0.100$ & $1.786 \pm 0.152$ \\
\hline
\end{tabular}

\subsection{Transcriptome Sequencing and De Novo Assembly}

To acquire a comprehensive overview of $A$. megalophylla, cDNA libraries were constructed from leaf samples of A. megalophylla harvested in May, August, and October. The libraries were sequenced using Illumina paired-end sequencing technology. After stringent data filtering and quality assessment, approximately 44.2, 58.5, and 47.3 million clean reads were obtained for the transcriptomes in May, August, and October, respectively (Table 2). Then, all the high-quality clean reads were de novo assembled using the Trinity program. A total of 83,886 unigenes, which comprised $68,294,276 \mathrm{bp}$, were acquired, with an average length of $814 \mathrm{bp}$, a maximum length of 17,500 bp, and an N50 of $1648 \mathrm{bp}$ (Table S1). The Q30 values and Q20 values were greater than $95.40 \%$ and $91.12 \%$, respectively, and GC content ranged from $45.20 \%$ to $46.75 \%$ (Table 2). The size distribution of these generated unigenes is shown in Figure S1; 74.45\% of all unigenes showed lengths ranging from 200 to $1000 \mathrm{bp}$, unigenes longer than $1000 \mathrm{bp}$ in length accounted for $24.45 \%$ of the total, and unigenes shorter than $200 \mathrm{bp}$ accounted for the remaining $1.1 \%$.

Table 2. Summary of transcriptomes from leaf in A. megalophylla.

\begin{tabular}{ccccccc}
\hline Item & Sample & Read Number & Base Number (bp) & GC Content & Q20 & Q30 \\
\hline \multirow{2}{*}{ Raw data } & S-5 & $44,211,695$ & $5,526,461,833$ & $45.83 \%$ & $95.31 \%$ & $91.25 \%$ \\
& S-8 & $58,464,607$ & $7,308,075,917$ & $46.19 \%$ & $95.75 \%$ & $92.07 \%$ \\
& S-10 & $47,325,290$ & $5,915,661,250$ & $46.17 \%$ & $95.10 \%$ & $90.86 \%$ \\
Clean data & S-5 & $43,135,219$ & $5,391,902,417$ & $45.88 \%$ & $95.74 \%$ & $91.71 \%$ \\
& S-8 & $57,752,125$ & $7,219,015,583$ & $46.23 \%$ & $96.13 \%$ & $92.46 \%$ \\
& S-10 & $45,535,950$ & $5,691,993,750$ & $46.15 \%$ & $95.51 \%$ & $91.32 \%$ \\
\hline
\end{tabular}

\subsection{Functional Annotation and Classification of A. megalophylla Unigenes}

After de novo assembly, 83,886 unigenes were generated. There were differences in the quantity and expression pattern of these unigenes among libraries from samples collected during different months. A total of 28,656 unigenes were expressed in all three months, while 16,379, 10,008, and 11,860 unigenes were expressed specifically in May, August, and October, respectively (Figure S2). The unigenes generated in this study were searched against the following public protein databases: NCBI-NR (National Center for Biotechnology Information-non-redundant protein database), Swiss-Prot, GO (Gene Ontology), Pfam, EggNOG (Evolutionary genealogy of genes: Non-supervised Orthologous Groups), KEGG (Kyoto Encyclopedia of Genes and Genomes), TmHMM (transmembrane protein prediction), and SignalP databases. Among 83,886 unigenes, a total of 50,054 (59.67\%) unigene sequences were annotated in the eight public databases. Between them, 41,139 $(49.04 \%), 38,096$ (45.41\%), 22,788 (27.17\%), 19,906 (23.73\%), 10,863 (12.95\%), 12,639 (15.07\%), 8860 (10.56\%), and 2967 $(3.54 \%)$ of the unigenes presented matches in the Swiss-Prot, Blast-NR, GO, Pfam, EggNOG, KEGG, TmHMM, and SignalP databases, respectively (Table 3).

$\mathrm{GO}$ annotation was used for classifying the function of unigenes. A total of 22,788 unigenes were grouped into three main functional categories and were then further classified into 52 subgroups, including 14 terms of cellular components (CC), 14 terms of molecular functions (MF), and 24 terms of biological processes (BP). In the CC, a high percentage of unigenes were linked with the terms "cell", "cell part", "orangelle", and "orangelle part". Most of the GO terms related to MF unigenes were "binding" and "catalytic activity", followed by "structural molecule" and "transcription regulator". 
Most of the assignments in BP were "cell process", "metabolic process", "biological regulation", and "response to stimulus" (Figure 2).

Table 3. Summary of the annotations for the assembled unigenes in public databases.

\begin{tabular}{ccc}
\hline Category & Account & Mapped Ratio (\%) \\
\hline Annotated & 50,054 & $59.67 \%$ \\
Swiss-Prot & 41,139 & $49.04 \%$ \\
Blast-NR & 38,096 & $45.41 \%$ \\
GO & 22,788 & $27.17 \%$ \\
Pfam & 19,906 & $23.73 \%$ \\
KEGG & 12,693 & $15.07 \%$ \\
EggNOG & 10,863 & $12.95 \%$ \\
TmHMM & 8860 & $10.56 \%$ \\
SignalP & 2967 & $3.54 \%$ \\
All & 83,886 & $100.00 \%$ \\
\hline
\end{tabular}

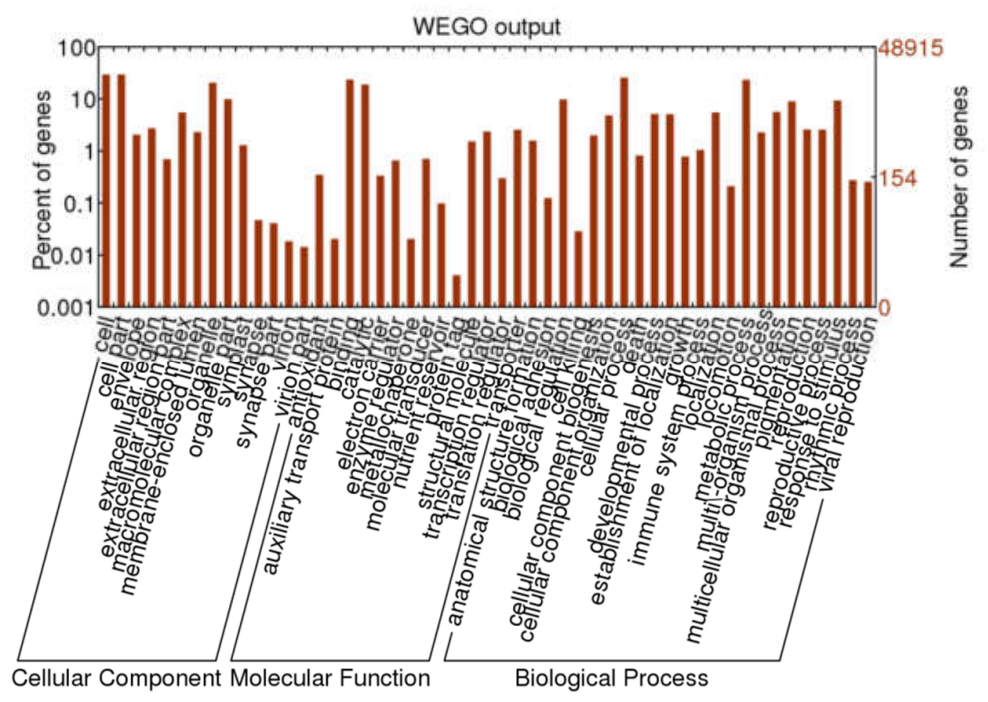

Figure 2. Gene ontology classification of A. megalophylla unigenes.

To further determine biochemical pathways, all the unigenes were searched against the KEGG database, and a total of 12,639 entries were annotated. These were classified into five main groups. Among them, the pathways most represented were "metabolism" (7092 unigenes), followed by "genetic information processing" (3170 unigenes), "organismal systems" (2027 unigenes), "cellular processing" (1515 unigenes), and "environmental information processing" (1345 unigenes) (Figure 3). This annotation information could aid further investigation of specific processes, functions, and pathways in A. megalophylla.

Transcription factors (TFs) play an important regulatory role in plant functions such as growth, development, and metabolism. Many TF families consist of diverse groups of gene families, including MYB, MYB-related, bHLH, AP2-EREBP, C2H2, and others. Results from our transcriptome data revealed that 1458 transcripts encode potential TFs, which can be sorted into $47 \mathrm{TF}$ families (Figure 4). Members of the MYB and MYB-related transcription factors were the most abundant $(206,14.13 \%)$ followed by bHLH (94, 6.45\%), AP2-EREBP $(91,6.24 \%)$, and C2H2 $(72,4.94 \%)$. The identification of TFs provided more abundant information to characterize TFs in various biochemical pathways in A. megalophylla. 


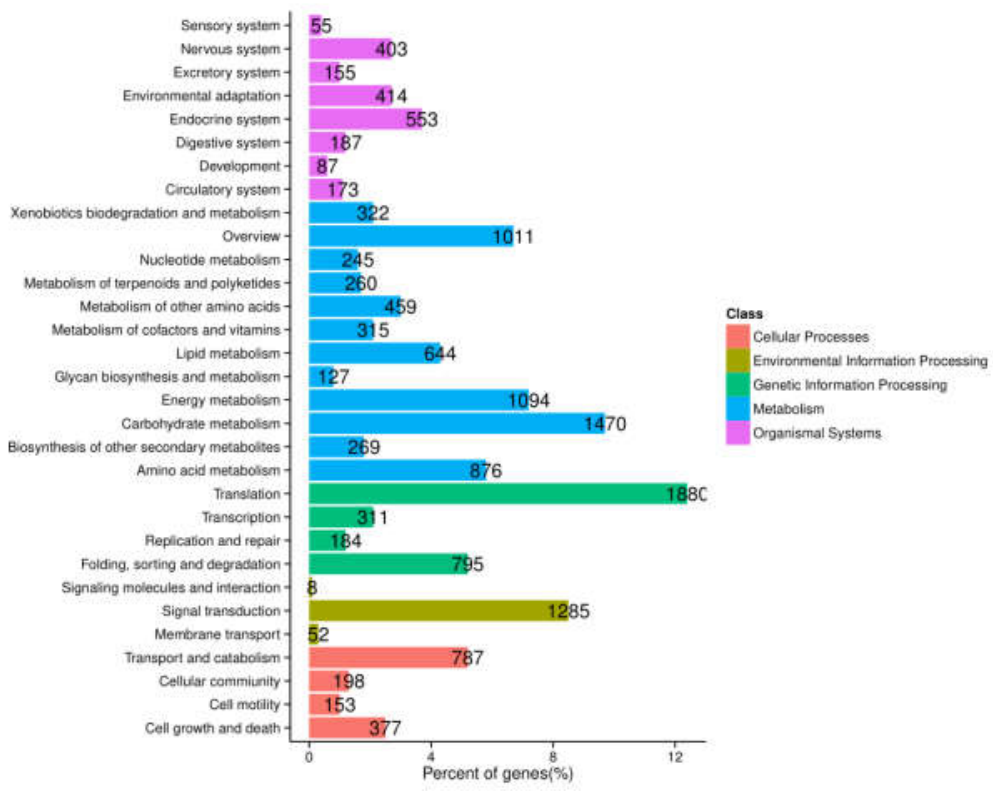

Figure 3. Functional classification and pathway assignment of assembled unigenes by KEGG.

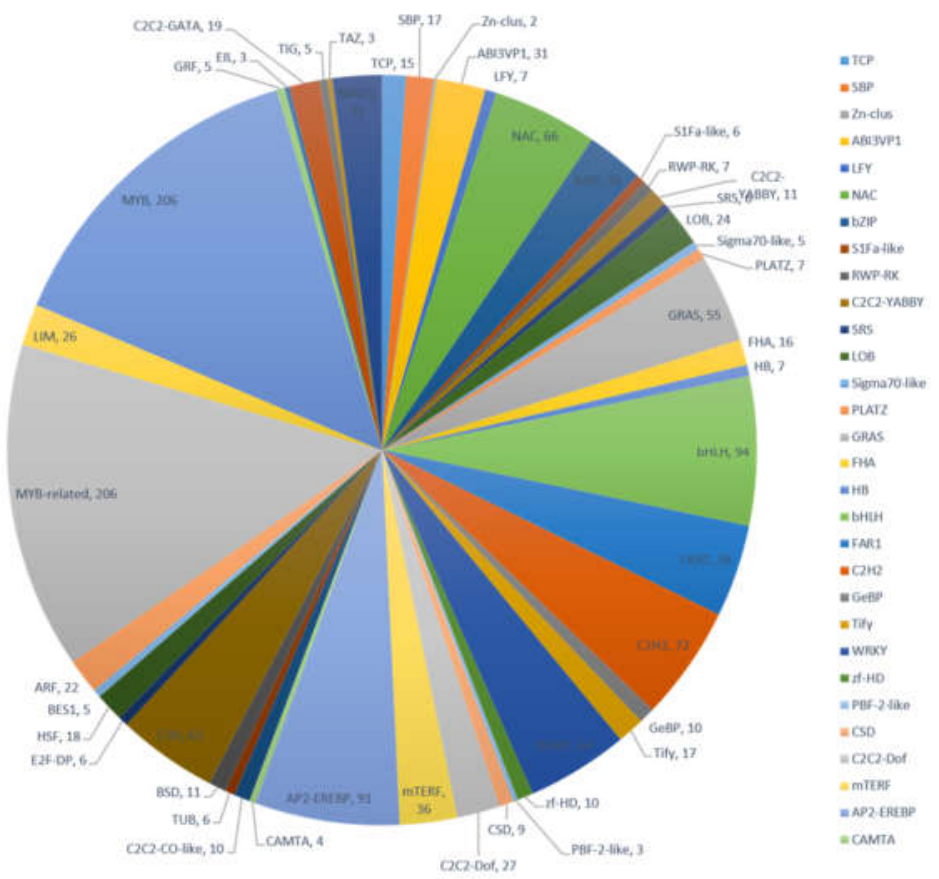

Figure 4. Distribution of transcription factors in A. megalophylla.

\subsection{Differentially Expressed Gene (DEG) Analysis of A. megalophylla}

We explored the transcriptomes of samples to identify differentially expressed genes (DEGs) during different months. Transcripts with $>\log$ two-fold differential expression and with false discovery rates (FDRs) of $>0.05$ were used as a threshold for the DEG analysis. There were 9096 DEGs between the May and August libraries, of which 3905 were up-regulated in May and 5191 were up-regulated in August. There were 10,613 DEGs identified between the May and October libraries, of which 3747 were up-regulated in May and 6866 were up-regulated in October. There were 393 DEGs between the August and October libraries, of which 64 were up-regulated in August and 329 were up-regulated in October (Figure 5). 
A

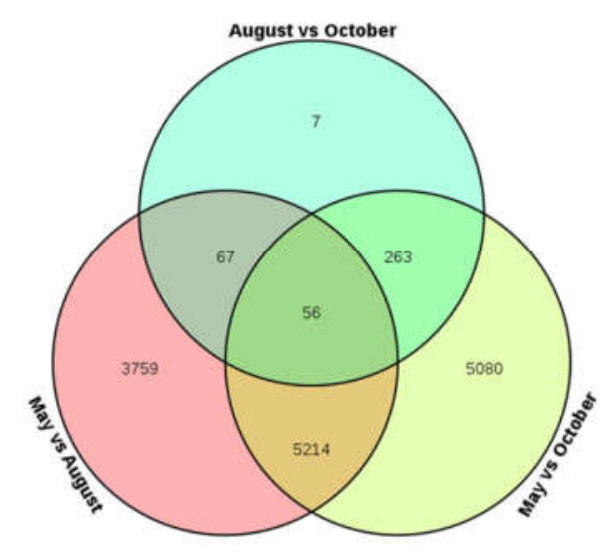

B

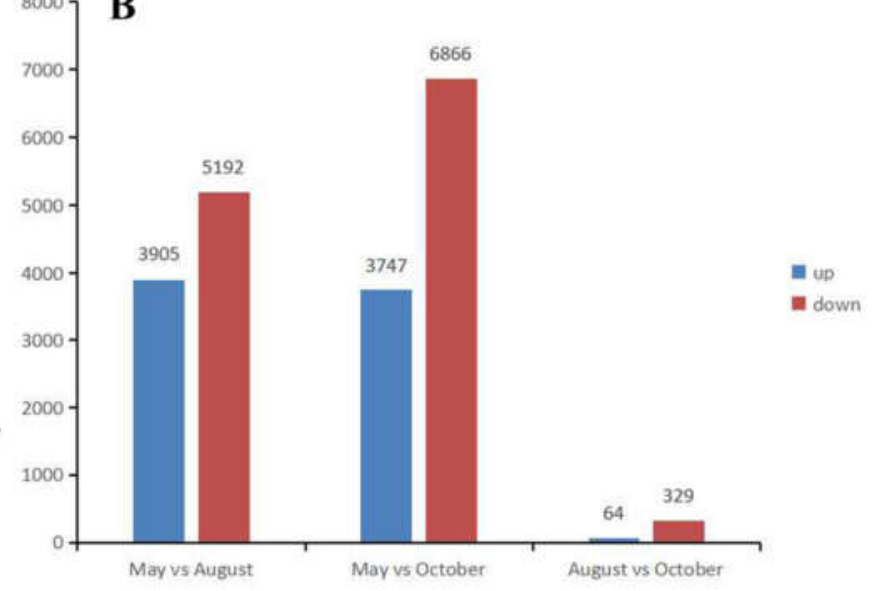

Figure 5. (A) The Venn diagram of the number of differentially expressed genes (DEGs); (B) The number of up-regulated and down-regulated DEGs.

To elucidate the functions and biological pathways of the DEGs, GO analysis and KEGG pathway analysis were performed. In the GO enrichment analysis, nearly half of the DEGs were assigned to the term "biological process". Among them, genes linked with the terms "cell process", "metabolic process", "response to stimulus", and "biological regulation" were highly enriched. Within the "cellular component" enriched set, genes linked to the terms "cell", "cell part", "organelle", and "organelle part" were highly enriched. On the other hand, GO terms related to "molecular function", such as "binding", "catalytic", "structural molecule", and "transcription regulator" were highly enriched depending on the month (Figure S3).

In the KEGG analysis, among 9097 DEGs of the 'May vs. August' comparison, 4092 unigenes were mapped into 303 KEGG pathways. There were 301 pathways in the 'May vs. October' comparison and 85 pathways in the 'August vs. October' comparison. Among these, pathways involved in the cell cycle, various metabolic pathways, and secondary metabolite biosynthesis, such as phenylpropanoids, flavone, and flavonol flavonoid biosynthesis, were significantly enriched.

\subsection{Candidate Genes Related to Flavonoid Biosynthesis}

Flavonoids were the main active compounds found in A. megalophylla and were important for evaluating its quality. To understand the regulation mechanisms of flavonoid biosynthesis in A. megalophylla, key regulatory genes of flavonoid biosynthesis involved in the pathways for phenylpropanoid biosynthesis, flavonoid biosynthesis, and flavone and flavonol biosynthesis were identified in this study. Furthermore, their expression profiles were comprehensively analyzed to assess seasonal variation in expression. The assumed flavonoid synthesis pathway is shown in Figure 6 .

Thirty-one unigenes encoding 15 key enzymes observed in this study were mostly related to biosynthesis of flavonoids, including anthocyanidin synthase (ANS, EC: 1.14.20.4), flavanone 3-hydroxylase ( $F 3 H$, EC: 1.14 .11 .9$)$, flavonoid-3' $5^{\prime}$-hydroxylase $\left(F 3^{\prime}, 5^{\prime} H\right.$, EC: 1.14 .14 .81$)$, caffeoyl-CoA O-methyltransferase (CCOMT, EC: 2.1.1.104), dihydroflavonol 4-reductase (DFR, EC: 1.1.1.219), 4-coumarate-CoA ligase (4CL, EC: 6.2.1.12), phenylalanine ammonia-lyase (PAL, EC: 4.3.1.24), flavonoid 3-methyltransferase (F3MT, EC: 2.1.1.76), cinnamate 4-hydroxylase (C4H, EC: 1.14.14.91), flavonoid $3^{\prime}$-monooxygenase ( $F 3^{\prime} H$, EC: 1.14 .14 .82$)$, chalcone synthase (CHS, EC: 2.3.1.74), shikimate O-hydroxycinnamoyltransferase (HCT, EC: 2.3.1.133), leucoanthocyanidin reductase (LAR, EC: 1.17.1.3), caffeic acid 3-O-methyltransferase (COMT, EC: 2.1.1.68), and flavonoid $3^{\prime}, 5^{\prime}$-methyltransferase $\left(F 3^{\prime}, 5^{\prime} M T\right.$, EC: 2.1.1.267) (Table S2). Furthermore, expression pattern analysis showed that these genes were expressed quite differently during different months (Figure 7). In particular, 18 DEGs, including CHS, PAL, F3H, F3', $5^{\prime} H, C 4 H, F 3^{\prime} H, A N S, F 3^{\prime}, 5^{\prime} M T$ and $F 3 M T$, had the highest expression in May, whereas expression of nine DEGs, including $L A R, H C T$, and DFR, was highest in October. 


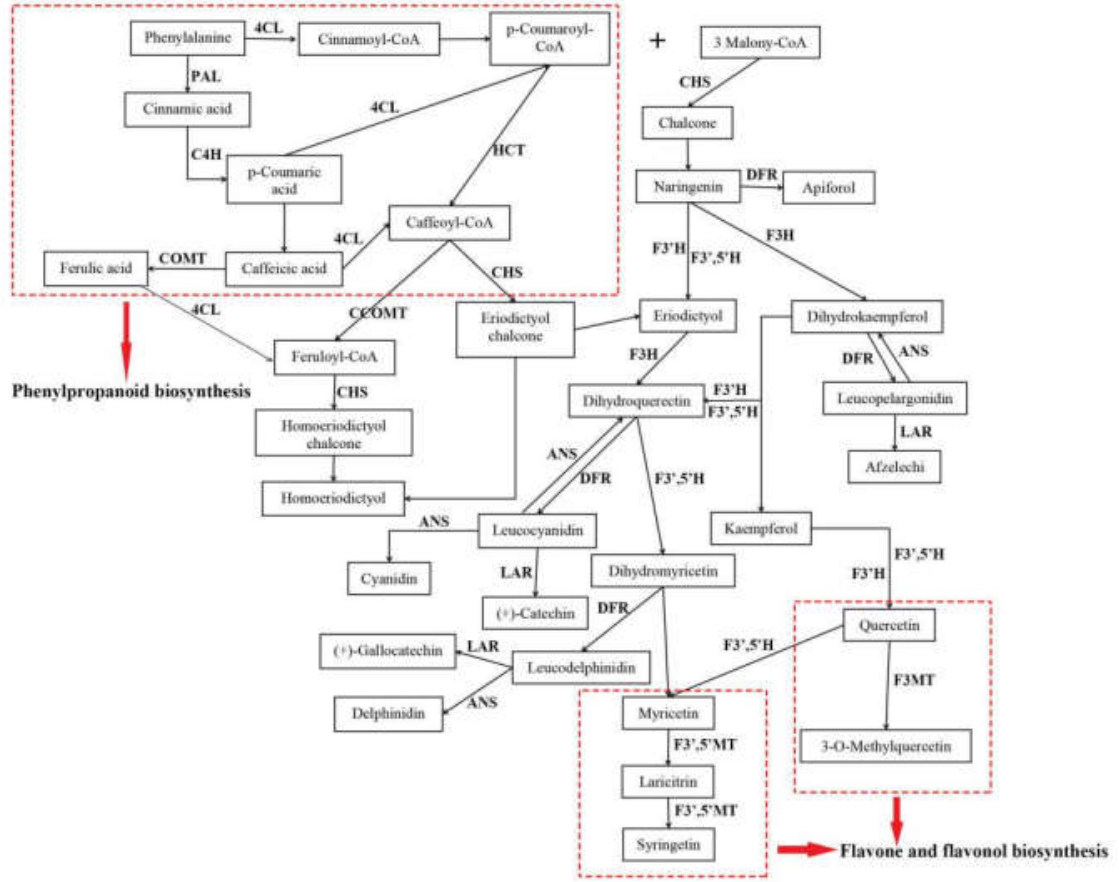

Figure 6. The flavonoid biosynthesis in A. megalophylla.

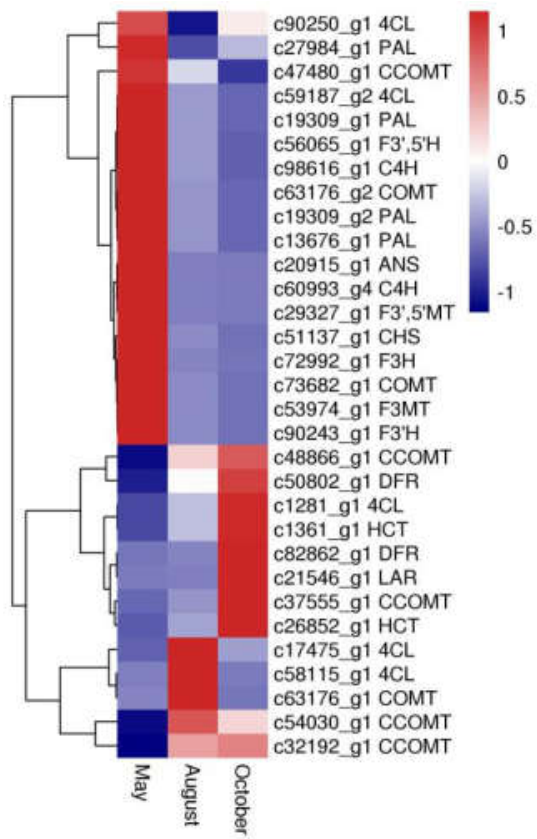

Figure 7. The expression patterns of DEGs involved in flavonoid biosynthesis. Blue and red colors represent different levels of gene expression and the color scales reflect a $\log _{2}$-transformed mean of fragments per kilobase of transcript per million mapped (FPKM) values.

\subsection{Candidate Genes Related to Flavonoid Accumulation and Biomodification}

The diversity of flavonoids and their distribution in plants depend not only on the expression patterns of key genes in the flavonoid biosynthesis pathway, but also on biomodification, transportation, and accumulation processes. The ATP binding cassette (ABC) transporter, soluble $N$-ethylmaleimide-sensitive factor attachment protein receptors (SNARE), and glutathione S-transferase (GST) have characteristic roles in flavonoid transportation and accumulation [10]. 
In our study, $50 \mathrm{ABC}$ transporter unigenes, 9 SNARE unigenes, and 49 GST unigenes were detected. The expression of most of the $\mathrm{ABC}$ transporter unigenes was highest in October and lowest in August. Most unigenes of both SNARE and GST had the highest expression in August and October, whereas only a few unigenes had the highest expression in May (Figure 8). Glycosyltransferases (GTs) may also have a vital function for flavonoid accumulation and biomodification. Analysis of the annotation data revealed 84 unigenes for glycosyltransferases. Among these genes, 29, 47, 5, and 3 unigenes were annotated to UDP-glycosyltransferases (UGTs), glucosyltransferases, mannosyltransferases and xylosyltransferases, respectively. The expression patterns of the GTs are shown in Figure 9. The glucosyltransferases and UDP-glycosyltransferases had the same expression levels. Most of the genes were highly expressed in August or October, whereas genes encoding mannosyltransferases and xylosyltransferases were highly expressed in May.
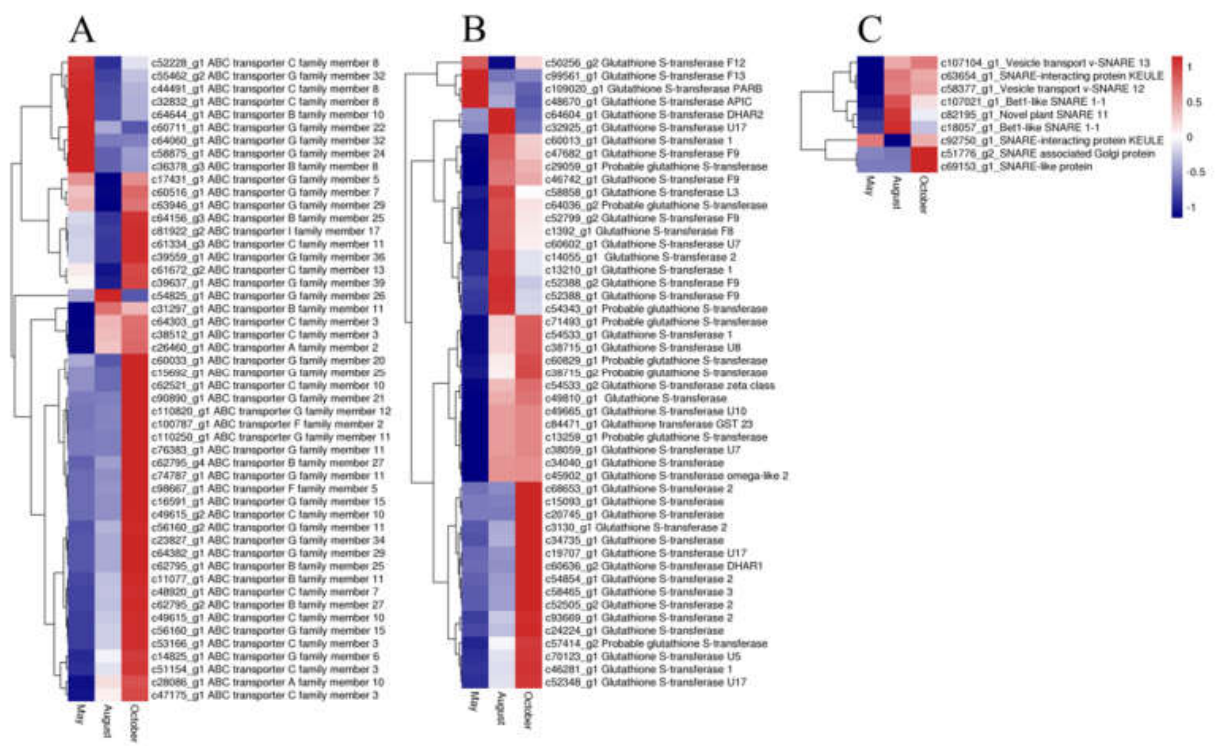

Figure 8. The expression level of DEGs of ATP binding cassette (ABC) transporter (A), glutathione S-transferase (GST) (B), and soluble $\mathrm{N}$-ethylmaleimide-sensitive factor attachment protein receptor (SNARE) (C) genes. Blue and red colors represent different levels of gene expression and the color scales reflect a $\log _{2}$-transformed mean of FPKM values.
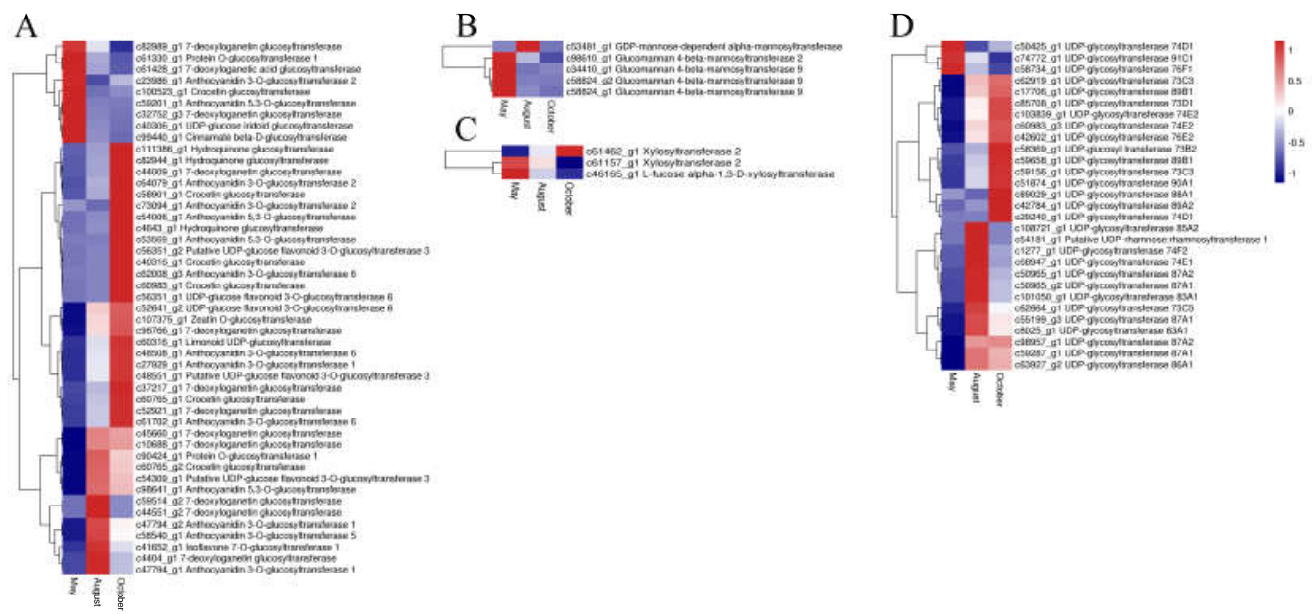

Figure 9. The expression level of glycosyltransferase DEGs including glucosyltransferases (A), mannosyltransferases (B), xylosyltransferases (C), UDP-glycosyltransferases (D). Blue and red colors represent different levels of gene expression and the color scales reflect a $\log _{2}$-transformed mean of FPKM values. 
2.7. Expression of Transcription Factors and P450 Family Genes Related Flavonoid Biosynthesis and Transport

Several flavonoid biosynthesis and transport genes are known to be transcriptionally regulated by TFs belonging to the MYB, bHLH (basic helix-loop-helix), and WD40 families $[12,13]$. These proteins usually act as a MYB-bHLH-WD40 (MBW) complex to regulate the biosynthetic pathway of flavonoids. In our analysis, five DEGs were annotated to MYB, two DEGs were annotated to bHLH, and three DEGs encoded WD40 repeat proteins. The expression levels or patterns of these three proteins are shown in Figure 10.

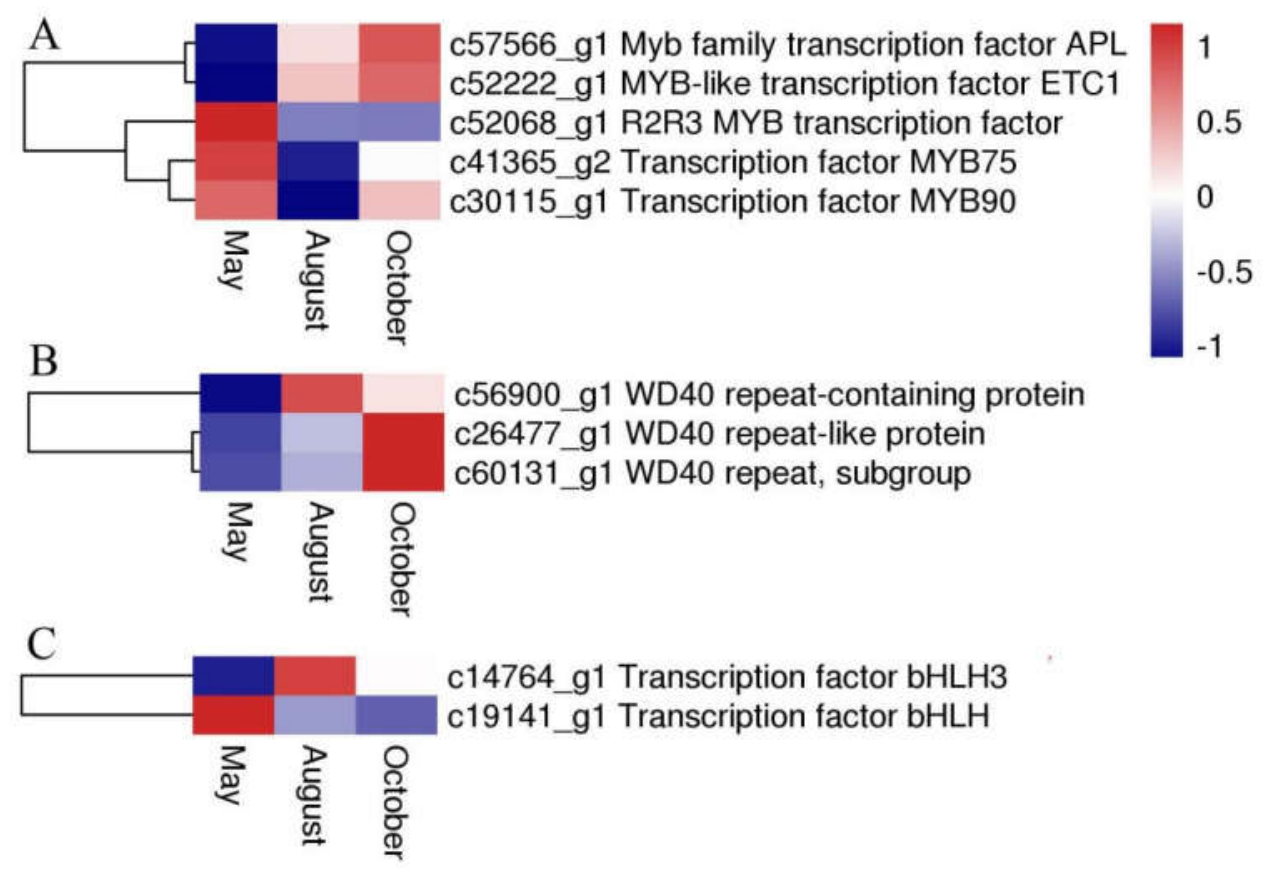

Figure 10. The expression level of DEGs encoding MYB (A), WD40 (B), and bHLH (C). Blue and red colors represent different levels of gene expression, and the color scales reflect a $\log _{2}$-transformed mean of FPKM values.

Cytochrome P450s (CYP450s), which comprise one of the largest and oldest gene superfamilies, are known to be involved in the biosynthesis of secondary metabolites such as flavonoids. In this study, 78 unigene DEGs were annotated as CYP450s. Most of these genes belonged to CYP71 (10 genes), nine of them belonged to CYP82, seven of them belonged to CYP704, six of them belonged to CYP86, and four of them belonged to CYP89. The expression levels of the P450 genes among the three months are shown in Figure 11. Sixteen P450 genes were most highly expressed in May, 42 P450 genes were most highly expressed in August, and twenty P450 genes were most highly expressed in October. 


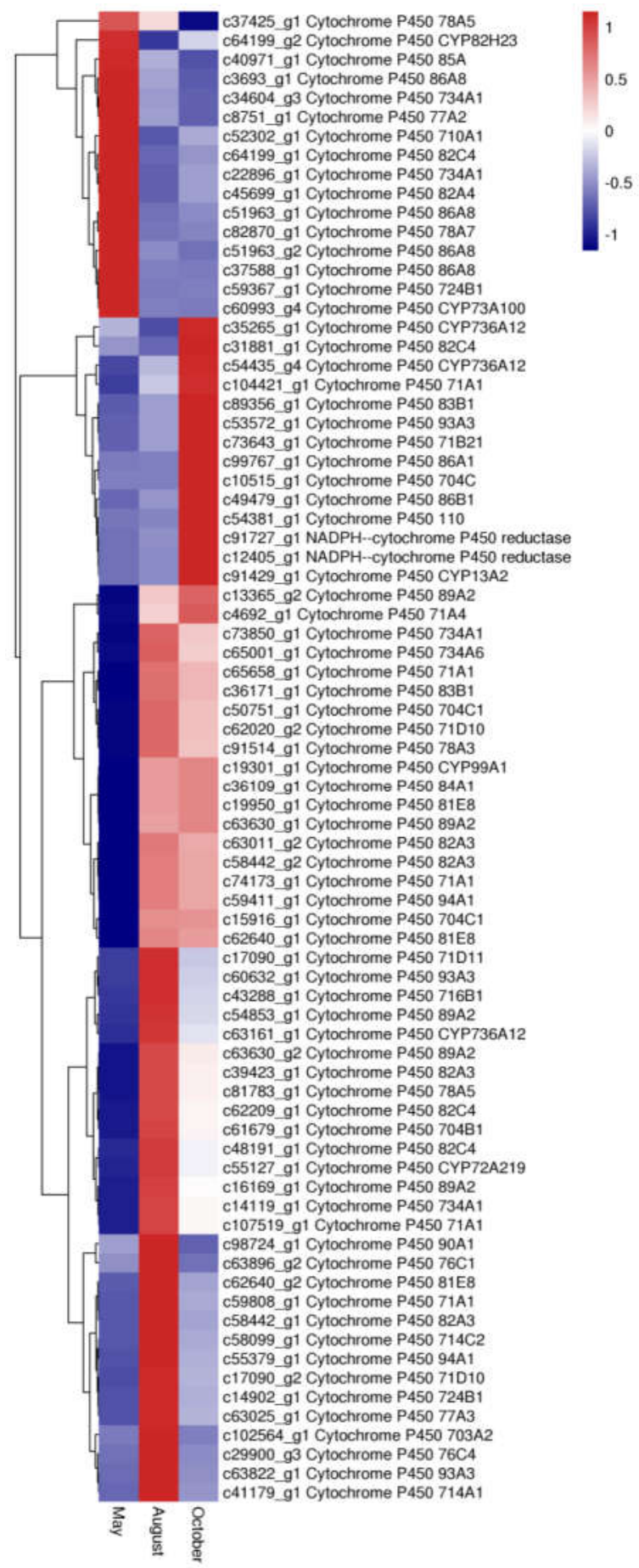

Figure 11. The expression level of DEGs of Cytochromes P450 in A. megalophylla. Blue and red colors represent different levels of gene expression, and the color scales reflect a $\log _{2}$-transformed mean of FPKM values.

\subsection{Validation of DEGs by $q P C R$}

To verify the accuracy of RNA-seq and computational analysis, five DEGs were selected randomly for qRT-PCR. The specific primers used for this analysis are shown in Table S3. All the genes selected for qRT-PCR analysis and those derived from sequencing had a similar trend in expression levels. In addition, correlation analysis between RNA-Seq and transcriptional data of these genes showed a positive correlation $\left(R^{2}=0.874\right)$ (Figure 12). 


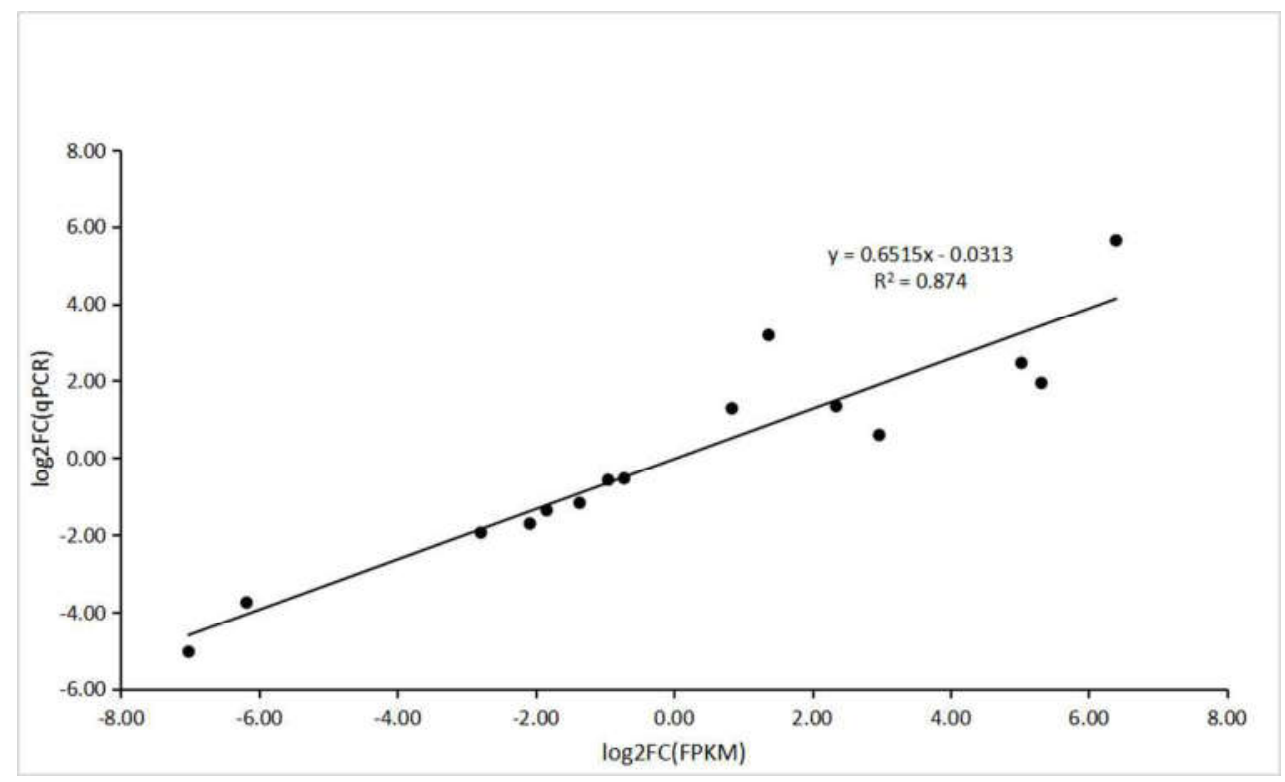

Figure 12. The correlation analysis between RNA-seq and qRT-PCR.

\section{Discussion}

The plant genus Ampelopsis contains about 30 species in the world and 17 species in China [14]. Most of them have medicinal value and biological activity because of their many active ingredients [14]. Within the Ampelopsis genus, A. megalophylla has been used as folk medicine for hundreds of years in China. Flavonoids are the most important active constituents in A. megalophylla. Flavonoids determine the quality of the plants, and flavonoid content varies across the season. However, the molecular mechanism of flavonoid biosynthesis and accumulation that causes seasonal variation in A. megalophylla flavonoid content remains largely unexplored. In the present study, we collected A. megalophylla samples during three different months and detected quantitative differences in flavonoids between the samples. The results showed that flavonoid contents in samples harvested in different months were vastly different from one another. The characterized flavonoids, including dihydromyricetin, myricitrin, and quercitrin, were found in greatest concentrations in May, followed by August, and were found in the lowest concentrations in October. To explore the reasons for these differences, RNA-Seq technology was used. In transcriptional analysis, a large number of transcripts exhibited a season-specific response. The number of DEGs in the 'May vs. October' and 'May vs. August' comparisons was much higher than that in the 'May vs. October' comparison. These results may imply that biological functions of $A$. megalophylla were extremely active in May.

Various key genes involved in regulation of flavonoid biosynthesis in higher plants have been reported $[15,16]$. The phenylpropanoid biosynthesis pathway (PATH: ec00940, KEGG) is the foundation to the synthesis of flavonoids, flavones, and flavonols. Cinnamoyl-CoA and 4-cinnamoyl-CoA Coumarin are the precursors of flavonoid synthesis. Their biosynthesis is regulated by related enzymes, such as $P A L, C 4 H$, and $4 C L[17,18]$, in phenylpropanoid pathways. Subsequently, chalcone is formed from 4-coumaroyl-CoA by CHS. This process is recognized as the first rate-limiting step in flavonoid biosynthesis [19]. After this step, different flavonoid subgroups are produced through modifying the molecular backbone, which is controlled by enzymes like $C H S, F 3 H, F 3^{\prime} H, F 3^{\prime}, 5^{\prime} H$, F3MT, F3', 5'MT, LAR, DFR, ANS, COMT, CCOMT, and HCT, which belong to phenylpropanoid biosynthesis, flavonoid biosynthesis (PATH: ec00941, KEGG), and flavone and flavonol biosynthesis (PATH: ec00944, KEGG) [16,17]. For example, methylation of flavonoids by $F 3 M T$ and $F 3^{\prime}, 5^{\prime} M T$, compared with nonmethylated flavonoids, can increase their metabolic stability, oral bioavailability, and biological activities [17]. In this work, homologous unigenes of the above genes were identified (Table S2). We also investigated the expression levels of these genes. Interestingly, DEGs encoding PAL, 
$C 4 H, C H S, F 3 H, F 3^{\prime}, 5^{\prime} H, F 3^{\prime} H, A N S, F 3^{\prime}, 5^{\prime} M T$, F3MT were more highly expressed in the samples from May than those from the other two months, and the highest flavonoid contents were also in the samples from May. This suggests that flavonoid biosynthesis in A. megalophylla may be significantly correlated with expression of these enzymes. It is noteworthy that in addition to high expression of $\mathrm{CHS}$, expression levels of $F 3^{\prime}, 5^{\prime} H, F 3 H$ were also found to be high in May. It has been previously reported that $\mathrm{F} 3 \mathrm{H}$ catalyzes eriodictyol into dihydroquercetin, which is the precursor of dihydromyricetin [20], and $F 3^{\prime}, 5^{\prime} H$ converts dihydroquercetin into dihydromyricetin [21], which is a main constituent in A. megalophylla. Therefore, $F 3 H$ and $F 3^{\prime}, 5^{\prime} H$ may be specific genes for dihydromyricetin synthesis in A. megalophylla.

The biosynthesis process in plants is sophisticated. Apart from the flavonoid biosynthesis pathway, there are also some genes involved in the biomodification, transportation, and accumulation processes of flavonoids. These genes include SNARE, ABC transporter, GST, GTs, CYP450, and the MYB-bHLH-WD40 complex.

$\mathrm{ABC}$ transporters are considered to be involved in the secretion of (iso)flavonoids from soybean roots and may regulate isoflavonoid levels in legumes [22]. SNARE proteins generate energy required for membrane fusion and participate in vesicle-mediated secretion during exocytosis and endocytosis [23]. GST protein, an important flavonoid transporter, is believed to be associated with transportation and separation of anthocyanins in vacuoles [24,25]. These genes were found in the present study and showed different expression patterns. Further study is warranted on the relationship between the effects of ABC transporter, SNARE, and GST gene expression on flavonoid content.

GTs are responsible for the glycosylation process, an important part of the flavonoid modification process, which is essential for producing tremendous chemical diversity and stable accumulation of flavonoids [26-29]. The ectopic expression of an Arabidopsis glycosyltransferase gene UGT76E11 resulted in increased flavonoid accumulation and tolerance to abiotic stresses in transgenic plants [30]. In addition, the biosynthesis and chemical diversity of flavonoid glycosides partly depended on UGTs [31,32]. Among the 29 UGTs in the present study, 26 unigenes were up-regulated in August and October and 3 unigenes were up-regulated in May. Because flavonoid content was highest in May, this suggests that the latter three unigenes were related to flavonoid accumulation in A. megalophylla. UGTs are consistently found in plants and particular roles of the DEGs require further investigation.

Cytochrome P450s are known to play central roles in the evolution of metabolic complexity [33] and in the production of secondary metabolites, including flavonoid metabolites [34]. In Oroxylum indicum, 31 diverse full length CYP450 genes participate in the biosynthesis of baicalin, baicalein, and other special metabolites [17]. Two CYP450s (CfCYP93B and CfCYP706C) isolated from Coleus forskohlii are involved in the biosynthesis of flavonoids [35] and CYP93B isolated from hybrid Gerbera encodes flavone synthase II, which catalyzes flavanones into flavones [36]. The expression of CYP450s also has a significant effect on the content of flavonoids in plants [37]. In addition, they, together with other enzymes like CHS, $\mathrm{CHI}$, drive flavonoid metabolism and plant-specific diversity in a flavonoid metabolon organization [38]. However, further research is needed to understand the exact role of CYP genes involved in the biosynthesis of certain flavonoids. Finally, the ternary MYB-bHLH-WD40 (5 MYB, 2 bHLH, 3 WD40) complex responsible for regulating flavonoid metabolic pathways is well characterized and is highly conserved throughout the plant kingdom. This complex activates and regulates the downstream gene expression of flavonoid metabolism, promotes the biosynthesis of procyanidins, and regulates the biosynthesis of procyanidins at the transcriptional level $[39,40]$. Therefore, the MBW complex in A. megalophylla, like in other plant species, may also play important roles in controlling the biosynthesis and accumulation of flavonoids.

The findings of this study may provide valuable genetic resources for further studies on species diversity of A. megalophylla and lay the foundation for studying marker genes to select cultivation with high contents of flavonoids and improve plants on the basis of genome level using genetic engineering. Future studies should explore the specific roles of the genes involved in flavonoid biosynthesis and accumulation in plants 


\section{Materials and Methods}

\subsection{Plant Materials}

A. megalophylla were collected from Laifeng County, Enshi Tujia and Miao Autonomous Prefecture, Hubei Province, China $\left(29^{\circ} 36.32^{\prime} \mathrm{N}, 109^{\circ} 15.98^{\prime} \mathrm{E}\right)$ on 10 th May, 21 th August, and 14 th October. They were three materials of leaves from the same plant. The plant materials were identified by Prof. Xiuqiao Zhang (Hubei University of Chinese Medicine). The leaves of $A$. megalophylla were immediately frozen in liquid nitrogen and stored at $-80^{\circ} \mathrm{C}$ prior to processing.

\subsection{Extraction and Determination of Flavonoids in A. megalophylla}

The samples collected in May, August, and October were dried and ground into powder. Powder samples $(0.5 \mathrm{~g})$ were treated for $30 \mathrm{~min}$ by ultrasonic extraction after soaking in petroleum ether $(150 \mathrm{~mL})$ for $30 \mathrm{~min}$. The homogenates were filtered, and residues were immersed in $70 \%$ alcohol $(20 \mathrm{~mL})$ for $30 \mathrm{~min}$ and extracted with $70 \%$ alcohol by ultrasonic treatment for $30 \mathrm{~min}$. For each extract, $2 \mathrm{~mL}$ was dissolved in $3.0 \mathrm{~mL}$ of methanol and filtered through a $0.45 \mu \mathrm{m}$ microporous film. Then, $20 \mu \mathrm{L}$ of filtrate was run on a Dionex-P680 HPLC (Dionex, Pliening, Germany) system with an Agilent TC-C18 column $(250 \mathrm{~mm} \times 4.6 \mathrm{~mm}, 5 \mu \mathrm{m}$, Agilent Technologies Inc., Palo Alto, AR, USA). The mobile phase consisted of acetonitrile (A solvent) and $0.1 \%$ phosphate solution (B solvent), used according to the following gradient elution program: $0 \mathrm{~min}, 0 \% \mathrm{~A} / 90 \% \mathrm{~B} ; 15 \mathrm{~min}, 15 \% \mathrm{~A} / 85 \% \mathrm{~B} ; 45 \mathrm{~min}, 15 \% \mathrm{~A} / 85 \% \mathrm{~B}$, $10 \mathrm{~min}, 25 \% \mathrm{~A} / 75 \% \mathrm{~B} ; 15 \mathrm{~min}, 25 \% \mathrm{~A} / 75 \% \mathrm{~B}$ and $15 \mathrm{~min}, 10 \% \mathrm{~A} / 90 \% \mathrm{~B}$. The detection wavelength was set at $280 \mathrm{~nm}$, and flow rate was $1 \mathrm{~mL} / \mathrm{min}$. The chemical standards included dihydromyricetin (15092141), myricitrin (11060222), and myricetin (14101531), which were purchased from Shanghai Yuanye Biotechnology Co., LTD, Shanghai, China, and quercetrin (20140318), which was purchased from Shanghai Jinsui Biotechnology Co., LTD.

\subsection{RNA Sequencing and De Novo Assembly}

Total RNAs were extracted using RNAprep Pure Plant Kit (Tiangen, Beijing, China) following the manufacturer's instructions. The RNA concentration and quantity were analyzed using a Nanodrop 2000 (Thermo Fisher Scientific, Waltham, MA, USA) and an Agilent 2100 (Agilent Technologies Inc., Palo Alto, AR, USA). A Stranded Total RNA Library Prep Kit (Illumina, Inc., San Diego, AR, USA) was used for cDNA library construction and normalization. The cDNA library was sequenced by Illumina HiSeq 2000 platform (Illumina, Inc., San Diego, AR, USA). Raw reads were filtered to obtain clean reads by removing sequences with ambiguous base pair identifications (' $\mathrm{N}$ ') and removing low-quality sequences. Then, high-quality clean reads were assembled into unigenes using Trinity software (v.2.4.0, the Broad Institute, Cambridge, MA, USA).

\subsection{Functional Annotation and Sequence Analysis}

The functions of unigenes were annotated using BLAST alignment of the sequences against the Swiss-Prot protein database, NCBI-NR, KEGG, and EggNOG. GO annotation was made by Blast2GO program (v.2.5, BioBam Bioinformatics, Valencia, Spain). These sequences were further analyzed with TMHMM (transmembrane protein prediction) to predict their protein transmembrane regions, and with SignalP to predict signal peptides. The transcription factor (TF) families were identified using known plant transcription factors identified in the PlnTFDB database based on the annotation.

\subsection{Analysis of Unigene Differential Expression Genes (DEGs)}

The FPKM (fragments per kilobase of transcript per million mapped) method was used to normalize and calculate gene expression. The DEGs were screened using the DESeq $R$ package with the threshold false discovery rate (FDR) of $<0.05$ and the absolute value of $\log _{2}$ FoldChange $>1$. Singular enrichment analysis of DEGs was carried out to identify the enriched Gene Ontology terms in 
all comparative conditions with DEGs at a significance level of 0.05 . Then, function analysis of the DEGs was also performed using the KEGG pathway.

\subsection{Quantitative Real-Time PCR}

The transcription levels of five putative key enzyme genes were investigated by real-time PCR using an iCycler iQ Real-Time PCR Detection System (Bio-Rad Laboratories, Hercules, CA, USA ) and using the Quant One Step RT-PCR kit (SYBR Green) (Tiangen, Beijing, China) with three replicates. Amplification reactions were performed with the following program: $50{ }^{\circ} \mathrm{C}$ for $30 \mathrm{~min}, 95^{\circ} \mathrm{C}$ for $2 \mathrm{~min}$, followed by 40 cycles of $94{ }^{\circ} \mathrm{C}$ for $20 \mathrm{~s}, 55^{\circ} \mathrm{C}$ for $20 \mathrm{~s}$, and $68^{\circ} \mathrm{C}$ for $20 \mathrm{~s}$. The expression level was normalized to the internal control gene GAPDH and calculated as $2^{-\Delta \Delta C t}$. All amplification primers are provided in Table S3.

\subsection{Statistical Analysis}

The data were expressed as the mean \pm SD of the five putative key enzyme genes from A. megalophylla plants collected during different months, and the relative expressions were calculated using the $2^{-\Delta \Delta C t}$ method. Significance tests were evaluated by one-way ANOVA. The results were analyzed statistically for significance $(p<0.05)$ using IBM SPSS Statistics 22.0 software (IBM Corp., Armonk, NY, USA).

Supplementary Materials: The following are available online. Figure S1: Length distribution of unigenes from samples of A. megalophylla; Figure S2: Venn diagram of unigenes involved in the three different time of A. megalophylla; Figure S3: Gene ontology classification of DEGs in A. megalophylla; Table S1: The specific primers of qRT-PCR; Table S2: Assembly results of clean reads; Table S3: Details of DEGs related to biosynthesis of flavonoid.

Author Contributions: Conceptualization, M.Y. and P.Z.; Data curation, P.Z.; Formal analysis, P.Z., C.G., and G.D.; Funding acquisition, L.G. and X.Z.; Investigation, G.D.; Methodology, C.G.; Project administration, X.Z.; Supervision, L.G.; Writing—original draft, M.Y. and P.Z.; Writing—review and editing, L.G. and X.Z.

Funding: This research was funded by the National Natural Science Foundation of China, grant number: 31170335, the Application Foundation and Advanced Technology of Wuhan Science and Technology Bureau, grant number: 2018060401011308, and the Education Bureau of Hubei Province, China, grant number: Q20182003.

Conflicts of Interest: The authors declare no conflict of interest.

\section{References}

1. Chen, M.L.; Chen, X.J.; Li, G.; Zhang, X.Q. Ampelopsis megalophylla ethanol extract of experimental studies on inhibition effect on expression of HBsAg, HBeAg 2215 cells. Chin. J. Tradit. Med. Sci. Technol. 2014, 19, 329-330.

2. Yang, R.J.; Chen, X.J.; He, X.Y.; Rao, R.; Zhang, X.Q.; Liu, Y.W. Study on anti-HBV virus effect of petroleum ether extracts of Ampelopsis megalophylla in vitro. Lishizhen Med. Materia Med. Res. 2011, 22, 153-215.

3. Xie, X.F.; Wang, J.W.; Zhang, H.P.; Li, Q.X.; Chen, B.Y. Chemical composition, antimicrobial and antioxidant activities of essential oil from Ampelopsis megalophylla. Nat. Prod. Res. 2014, 28, 853-860. [CrossRef]

4. Cheng, P.; Gui, C.; Huang, J.; Xia, Y.; Fang, Y.; Da, G.Z.; Zhang, X.Q. Molecular mechanisms of ampelopsin from Ampelopsis megalophylla induces apoptosis in HeLa cells. Oncol. Lett. 2017, 14, 2691-2698. [CrossRef] [PubMed]

5. Xie, X.F.; Wang, J.W.; Zhang, H.P.; Li, Q.X.; Chen, B.Y. Characterization and antitumor activities of a water-soluble polysaccharide from Ampelopsis megalophylla. Carbohydr. Polym. 2015, 129, 55-61. [CrossRef] [PubMed]

6. Shen, W.; Zhang, X.J.; Zhang, X.Q.; Zhang, Y.W. Study on chemical constituents of Ampelopsis megalophylla. Lishizhen Med. Mater. Med. Res. 2010, 22, 866-867.

7. Zheng, G.H.; Wu, J.; Liu, D.; Qiao, M. HPLC for Fingerprint Analysis of Flavones from Meicha from Different Harvest Time. Chin. Arch. Tradit. Chin. Med. 2009, 27, 980-982.

8. Katsuyama, Y.; Funa, N.; Miyahisa, I.; Horinouchi, S. Synthesis of unnatural flavonoids and stilbenes by exploiting the plant biosynthetic pathway in Escherichia coli. Chem Biol. 2007, 14, 613-621. [CrossRef] 
9. Nabavi, M.S.; Samec, D.; Tomczyk, M.; Milella, L.; Russo, D.; Habtemariam, S.; Suntar, I.; Rastrelli, L.; Daglia, M.; Xiao, J.; et al. Flavonoid biosynthetic pathways in plants: Versatile targets for metabolic engineering. Biotechnol. Adv. 2018. [CrossRef]

10. Lei, Z.; Zhou, C.; Ji, X.; Wei, G.; Huang, Y.; Yu, W.; Luo, Y.; Qiu, Y. Transcriptome Analysis Reveals genes involved in flavonoid biosynthesis and accumulation in Dendrobium catenatum from Different Locations. Sci. Rep. 2018, 8, 6373. [CrossRef]

11. Liu, X.; Lu, Y.; Yuan, Y.; Liu, S.; Guan, C.; Chen, S.; Liu, Z. De novo transcriptome of Brassica juncea seed coat and identification of genes for the biosynthesis of flavonoids. PLoS ONE 2013, 8, e71110. [CrossRef] [PubMed]

12. Lepiniec, L.; Debeaujon, I.; Routaboul, J.M.; Baudry, A.; Pourcel, L.; Nesi, N.; Caboche, M. Genetics and biochemistry of seed flavonoids. Annu. Rev. Plant Biol. 2006, 57, 405-430. [CrossRef]

13. Zifkin, M.; Jin, A.; Ozga, J.A.; Zaharia, L.I.; Schernthaner, J.P.; Gesell, A.; Abrams, S.R.; Kennedy, J.A.; Constabel, C.P. Gene expression and metabolite profiling of developing highbush blueberry fruit indicates transcriptional regulation of flavonoid metabolism and activation of abscisic acid metabolism. Plant Physiol. 2012, 158, 200-224. [CrossRef] [PubMed]

14. Xia, Y. Study of Medicinal Plants of Ampelopsis by DNA Molecular Identification and Chemical Composition Analysis. Master's Thesis, Hubei University of Chinese Medicine, Wuhan, China, 2016.

15. Li, C.F.; Zhu, Y.; Yu, Y.; Zhao, Q.Y.; Wang, S.J.; Wang, X.C.; Yao, M.Z.; Luo, D.; Li, X.; Chen, L.; et al. Global transcriptome and gene regulation network for secondary metabolite biosynthesis of tea plant (Camellia sinensis). BMC Genom. 2015, 16, 560. [CrossRef] [PubMed]

16. Yue, J.; Zhu, C.; Zhou, Y.; Niu, X.; Miao, M.; Tang, X.; Chen, F.; Zhao, W.; Liu, Y. Transcriptome analysis of differentially expressed unigenes involved in flavonoid biosynthesis during flower development of Chrysanthemum morifolium 'Chuju'. Sci. Rep. 2018, 8, 13414. [CrossRef] [PubMed]

17. Deshmukh, A.B.; Datir, S.S.; Bhonde, Y.; Kelkar, N.; Samdani, P.; Tamhane, V.A. De novo root transcriptome of a medicinally important rare tree Oroxylum indicum for characterization of the flavonoid biosynthesis pathway. Phytochemistry 2018, 156, 201-213. [CrossRef] [PubMed]

18. Tao, X.; Fang, Y.; Huang, M.J.; Xiao, Y.; Liu, Y.; Ma, X.R.; Zhao, H. High flavonoid accompanied with high starch accumulation triggered by nutrient starvation in bioenergy crop duckweed (Landoltia punctata). BMC Genom. 2017, 18, 166. [CrossRef] [PubMed]

19. Tohge, T.; Yonekura-Sakakibara, K.; Niida, R.; Watanabe-Takahashi, A.; Saito, K. Phytochemical genomics in Arabidopsis thaliana: A case study for functional identification of flavonoid biosynthesis genes. Pure Appl. Chem. 2007, 4, 811-823. [CrossRef]

20. Han, Y.; Huang, K.; Liu, Y.; Jiao, T.; Ma, G.; Qian, Y.; Wang, P.; Dai, X.; Gao, L.; Xia, T. Functional Analysis of Two Flavanone-3-Hydroxylase Genes from Camellia sinensis: A Critical Role in Flavonoid Accumulation. Genes 2017, 8, 300. [CrossRef]

21. Sun, Y.; Huang, H.; Meng, L.; Hu, K.; Dai, S.L. Isolation and functional analysis of a homolog of flavonoid 3',5'-hydroxylase gene from Pericallis $\times$ hybrida. Physiol. Plant 2013, 149, 151-159. [CrossRef]

22. Sugiyama, A.; Shitan, N.; Yazaki, K. Involvement of a soybean ATP-binding cassette-type transporter in the secretion of genistein, a signal flavonoid in legume-Rhizobium symbiosis. Plant Physiol. 2007, 144, 2000-2008. [CrossRef] [PubMed]

23. Lipka, V.; Kwon, C.; Panstruga, R. SNARE-ware: The role of SNARE-domain proteins in plant biology. Annu. Rev. Cell Dev. Biol. 2007, 23, 147-174. [CrossRef]

24. Klein, M.; Martinoia, E.; Hoffmann-Thoma, G.; Weissenbock, G. A membrane-potential dependent ABC-like transporter mediates the vacuolar uptake of rye flavone glucuronides: Regulation of glucuronide uptake by glutathione and its conjugates. Plant J. 2000, 21, 289-304. [CrossRef] [PubMed]

25. Castellarin, S.D.; Matthews, M.A.; Di Gaspero, G.; Gambetta, G.A. Water deficits accelerate ripening and induce changes in gene expression regulating flavonoid biosynthesis in grape berries. Planta 2007, 227, 101-112. [CrossRef]

26. Yoshida, K.; Toyama, Y.; Kameda, K.; Kondo, T. Contribution of each caffeoyl residue of the pigment molecule of gentiodelphin to blue color development. Phytochemistry 2000, 54, 85-92. [CrossRef]

27. Cos, P.; Calomme, M.; Sindambiwe, J.B.; De Bruyne, T.; Cimanga, K.; Pieters, L.; Vlietinck, A.J.; Vanden, B.D. Cytotoxicity and lipid peroxidation-inhibiting activity of flavonoids. Planta Med. 2001, 67, 515-519. [CrossRef] [PubMed] 
28. Danielsen, A.G.; Liu, F.; Hosomi, Y.; Shii, K.; Roth, R.A. Activation of protein kinase C alpha inhibits signaling by members of the insulin receptor family. J. Biol. Chem. 1995, 270, 21600-21605. [CrossRef]

29. Moreira, M.R.; Kanashiro, A.; Kabeya, L.M.; Polizello, A.C.; Azzolini, A.E.; Curti, C.; Oliveira, C.A.; T-do Amaral, A.; Lucisano-Valim, Y.M. Neutrophil effector functions triggered by Fc-gamma and/or complement receptors are dependent on B-ring hydroxylation pattern and physicochemical properties of flavonols. Life Sci. 2007, 81, 317-326. [CrossRef]

30. Li, Q.; Yu, H.M.; Meng, X.F.; Lin, J.S.; Li, Y.J.; Hou, B.K. Ectopic expression of glycosyltransferase UGT76E11 increases flavonoid accumulation and enhances abiotic stress tolerance in Arabidopsis. Plant Biol. 2018, 20, 10-19. [CrossRef]

31. Nagatomo, Y.; Usui, S.; Ito, T.; Kato, A.; Shimosaka, M.; Taguchi, G. Purification, molecular cloning and functional characterization of flavonoid C-glucosyltransferases from Fagopyrum esculentum M. (buckwheat) cotyledon. Plant J. 2014, 80, 437-448. [CrossRef]

32. Yonekura-Sakakibara, K.; Fukushima, A.; Nakabayashi, R.; Hanada, K.; Matsuda, F.; Sugawara, S.; Inoue, E.; Kuromori, T.; Ito, T.; Shinozaki, K.; et al. Two glycosyltransferases involved in anthocyanin modification delineated by transcriptome independent component analysis in Arabidopsis thaliana. Plant J. 2012, 69, 154-167. [CrossRef] [PubMed]

33. Bak, S.; Beisson, F.; Bishop, G.; Hamberger, B.; Hofer, R.; Paquette, S.; Werck-Reichhart, D. Cytochromes p450. Arab. Book 2011, 9, e0144. [CrossRef] [PubMed]

34. Fujino, N.; Tenma, N.; Waki, T.; Ito, K.; Komatsuzaki, Y.; Sugiyama, K.; Yamazaki, T.; Yoshida, S.; Hatayama, M.; Yamashita, S.; et al. Physical interactions among flavonoid enzymes in snapdragon and torenia reveal the diversity in the flavonoid metabolon organization of different plant species. Plant J. 2018, 94, 372-392. [CrossRef] [PubMed]

35. Awasthi, P.; Gupta, A.P.; Bedi, Y.S.; Vishwakarma, R.A.; Gandhi, S.G. Mannitol Stress Directs Flavonoid Metabolism toward Synthesis of Flavones via Differential Regulation of Two Cytochrome P450 Monooxygenases in Coleus forskohlii. Front Plant Sci. 2016, 7, 985. [CrossRef] [PubMed]

36. Martens, S.; Forkmann, G. Cloning and expression of flavone synthase II from Gerbera hybrids. Plant J. 1999, 20, 611-618. [CrossRef]

37. Ayabe, S.; Akashi, T. Cytochrome P450s in flavonoid metabolism. Phytochem. Rev. 2006, 5, 271-282. [CrossRef]

38. Jin, M.; Zhu, Z.B.; Guo, Q.S.; Shen, H.J.; Wang, Y.R. Growth and accumulation of bioactive compounds in medicinal Chrysanthemum morifolium Ramat. cv. 'Chuju' under different colored shade polyethylene. J. Med. Plants Res. 2012, 6, 398-404. [CrossRef]

39. Schuler, M.A. The role of cytochrome P450 monooxygenases in plant-insect interactions. Plant Physiol. 1996, 112, 1411-1419. [CrossRef]

40. Peng, Y.; Lin-Wang, K.; Cooney, J.M.; Wang, T.; Espley, R.V.; Allan, A.C. Differential regulation of the anthocyanin profile in purple kiwifruit (Actinidia species). Hortic. Res. 2019, 6, 3. [CrossRef]

Sample Availability: Not available.

(C) 2019 by the authors. Licensee MDPI, Basel, Switzerland. This article is an open access article distributed under the terms and conditions of the Creative Commons Attribution (CC BY) license (http://creativecommons.org/licenses/by/4.0/). 